\title{
Les activités d'une salle de travaux pratiques informatisée
}

\author{
M. Tropis, C. Bouysset, N. Cauvin, Ch. Salle, A. Megel et Groupe Ideao
}

\section{(2) OpenEdition}

1 Journals

Édition électronique

URL : http://journals.openedition.org/trema/2378

DOI : $10.4000 /$ trema.2378

ISSN : 2107-0997

Éditeur

Faculté d'Éducation de l'université de Montpellier

\section{Édition imprimée}

Date de publication : 1 mai 1993

Pagination : 125-129

ISSN : 1167-315X

\section{Référence électronique}

M. Tropis, C. Bouysset, N. Cauvin, Ch. Salle, A. Megel et Groupe Ideao, «Les activités d'une salle de travaux pratiques informatisée », Tréma [En ligne], 3-4 | 1993, mis en ligne le 01 mai 1993, consulté le 01 mai 2019. URL : http://journals.openedition.org/trema/2378 ; DOI : 10.4000/trema.2378

Ce document a été généré automatiquement le 1 mai 2019.

Trema 


\title{
Les activités d'une salle de travaux pratiques informatisée
}

\author{
M. Tropis, C. Bouysset, N. Cauvin, Ch. Salle, A. Megel et Groupe Ideao
}

1 Les activités de modélisation utilisant l'ordinateur ou d'une manière plus générale les démarches de type «inductiviste » où l'élève est amené à concevoir une expérience, le traitement des mesures et leur interprétation dans le but de résoudre un problème expérimental, ont connu ces dernières années un foisonnement de publications où on les trouve abondamment décrites et hautement préconisées $(1,2)$.

2 Malheureusement, si elles ont pu être quelquefois pratiquées (3), elles ont subi trois inconvénients qui n'ont jamais permis de les réaliser jusqu'au bout :

- leur absence d'intégration dans le déroulement normal du cursus ; il s'agit souvent d'expériences faites par un enseignant qui n'a pas lui-même imaginé les situations, avec des élèves sélectionnés lors de cours facultatifs et à des périodes de l'année où le thème n'est pas du tout d'actualité (2) ;

- l'absence de matériel qui fait que l'activité n'est jamais pratiquée individuellement car il faudrait que chaque binôme possède un équipement complet ;

- le manque de temps qui fait que l'activité n'est jamais conduite à son terme. On voudrait que dans l'espace d'une séance de TP, l'élève puisse réfléchir au sujet, imaginer l'expérience, appréhender la machine et le logiciel, réaliser l'expérience, obtenir le modèle, faire fonctionner le modèle dans des situations différentes.

Dans la plupart de telles activités, on se contente d'une validation du modèle reconnu par l'institution et imposé par le professeur et cela même si la démarche est inductive (4).

4 Nous allons décrire une expérimentation qui a commencé au lycée de Manosque au printemps 1992. Cet établissement a l'avantage de posséder :

- une équipe de professeurs de sciences physiques formés à l'ordinateur outil de laboratoire depuis plusieurs années et qui utilisent depuis lors et régulièrement ces techniques dans leurs classes de seconde, première et terminale. Ces pratiques, de même que celles que nous allons décrire au sujet de l'expérimentation sont donc parfaitement intégrées au déroulement normal du programme scolaire et se font avec des classes complètes d'élèves non sélectionnés. Chaque professeur joue alternativement le rôle d'observateur. 
- un équipement à peu près unique en France constitué de deux salles de travaux pratiques informatisées.

5 La première salle obéit au concept de classe 2000 tel que nous l'avions défini il y a quelques années et qui a été largement présenté aux dernières journées Informatique et Pédagogie des Sciences Physiques de Marseille (5). Cette salle est équipée pour la réalisation de travaux pratiques collectifs avec visualisation de l'ordinateur sur grand écran. Dans l'optique de l'expérimentation cette salle est utilisée pour les séances collectives de confrontation des résultats individuels des élèves.

6 La deuxième salle est une salle de travaux pratiques ou chaque binôme d'élèves possède un système informatique complet soit un ordinateur, une imprimante, un système d'acquisition de données avec ses capteurs et le matériel d'expérience. Le poste professeur, également équipé permet de collecter les résultats des élèves pour confrontation, contrôle du travail ou activités collectives (par exemple exercices communs sur des données d'élèves). Il sert également pour accéder à des périphériques non disponibles sur les postes comme par exemple le traceur de courbes.

7 Les logiciels retenus pour l'expérimentation sont:

- pour les expériences d'électricité, le logiciel LABO de Langage et Informatique, spécialisé dans toute expérience pouvant être visualisée avec un oscilloscope,

- pour les expériences de mécanique, le logiciel DIGITAB de Pierron dédié a l'étude des mouvements sur table à coussin d'air.

Ces deux logiciels ont été conçus par des membres de l'équipe I.D.E.A.O.

Les thèmes scientifiques retenus pour l'expérimentation sont limités à trois : l'étude de la charge du condensateur, les circuits électriques dérivateurs et intégrateurs et le mouvement parabolique sur un plan incliné capté par l'intermédiaire d'une tablette à numériser $(6,7)$. Ces thèmes concernent tous la classe de première $S$.

10 Décrivons la démarche qui est demandée pour le circuit dérivateur. L'activité sera pratiquée dans un système de fonctionnement normal pour des travaux pratiques en lycée, c'est-à-dire que l'ensemble de la classe (ou de la demi-classe) est sensé traiter la même expérience pendant le même temps. On propose aux élèves de réaliser un circuit électrique précis et on leur demande de l'identifier. On pose comme hypothèse que lorsqu'on envoie un signal électrique en entrée du circuit, il donne en sortie un autre signal et que la réponse réside dans la comparaison des deux signaux. L'élève doit donc choisir quel type d'alimentation employer (continue, sinusoïdale, triangulaire, carrée), réaliser l'acquisition, utiliser les fonctions de calcul et de modélisation du logiciel pour traiter les courbes enregistrées et identifier la propriété.

11 Lorsque le résultat est obtenu, on essaie de lui faire extrapoler la propriété vers un autre cas, à savoir le circuit intégrateur, il doit donc imaginer la modification du circuit qu'il faut réaliser et effectuer la vérification de la propriété. Cette étude pourrait être faite avec un simple oscilloscope, on souhaite cependant que l'élève puisse entrer dans une conceptualisation mathématique afin de pouvoir en évaluer l'importance. L'élève devra obtenir une modélisation mathématique des signaux électriques et pourra utiliser différents aspects du concept de dérivée (étude qualitative simple, étude quantitative sur quelques points, passage par une formulation analytique, utilisation de la dérivation numérique). 
12 L'objectif de cette expérimentation ne concerne pas pour autant l'étude des modèles et concepts. Elle a pour but de répondre à un certain nombre de questions au sujet d'abord de l'attitude des élèves devant l'outil, ensuite des objectifs généraux de ces activités.

13 Au sujet de l'attitude des élèves, les questions concernent l'appréhension de l'outil, la connaissance du fonctionnement du système d'acquisition et des logiciels (surtout en ce qui concerne les méthodes de traitement numérique), les prérequis et la formation préalable nécessaire. Faut-il leur expliquer le fonctionnement ou considérer l'outil comme une boîte noire? Quelles peuvent être par exemple les questions des élèves lorsqu'ils voient un modèle théorique ajusté de par la valeur du khi2 ou du critère de confiance ou lorsqu'ils observent des courbes de dérivées numériques en dent de scie (8) ? Une question plus délicate concerne l'objectif général de ces pratiques. Doivent-elles être :

- un simple outil facilitant l'appropriation des concepts,

- une redécouverte des lois,

- une probation des lois,

- une initiation à la recherche,

- une formation à la recherche scientifique.

\section{En résumé}

Ces activités doivent-elles être de véritables activités scientifiques ou des transpositions didactiques, des pratiques scolaires traditionnelles ou des pratiques de référence?

Coupe verticale des corps de pompe et des pistons.

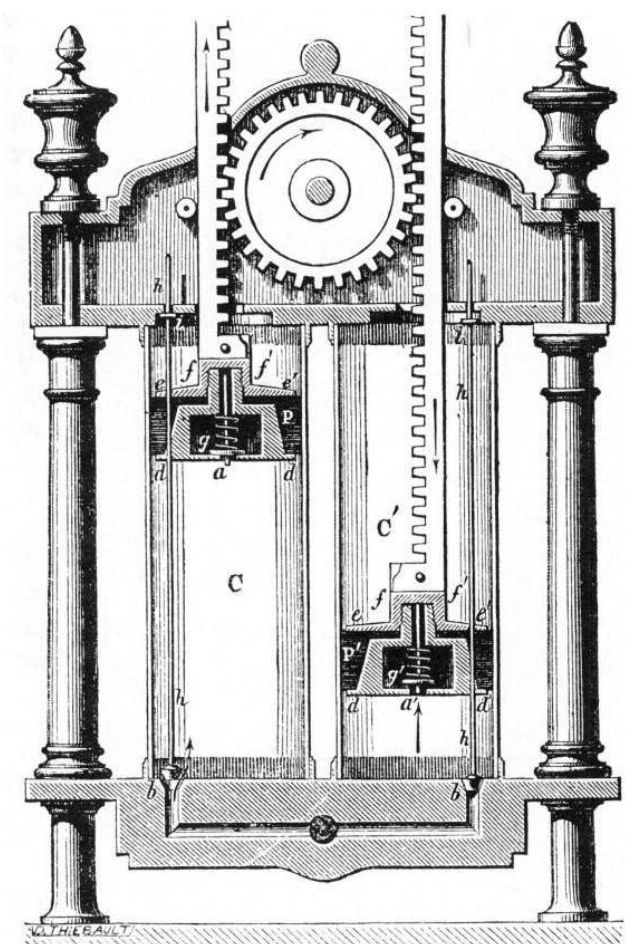




\section{BIBLIOGRAPHIE}

\section{Références}

1. C. BOUYSSET, R. KRAEMER et L.VIEL (1985). Réflexions sur les apports didactiques des systèmes d'acquisition de données dans l'enseignement des disciplines scientifiques. Actes du séminaire couplage de micro-ordinateurs avec travaux pratiques et périphériques multimédia. Toulouse.

2. A. DUREY (1987). Vers des activités didactiques de mise au point de modèles de physique avec des micro-ordinateurs. Thèse d'état. Paris.

3. JL. FIRPO. P. NEEL et J. BOYER (1989). Manipulations assistées par ordinateur. Actes des 11èmes journées internationales sur l'éducation scientifique. Chamonix.

4. A. GUILLON (1990). Modélisation, quelques questions. Actes des 4èmes journées IPSP Toulouse.

5. A. MEGEL (1992). Classe 2000. Actes des 5èmes journées IPSP. Marseille.

6. A. MEGEL (1990). Etude expérimentale du mouvement parabolique. Fiches pratiques d'utilisation de l'informatique en classe de physique et chimie. Editions UdP. INRP.

7. A. MEGEL (1990). La mécanique à coussin d'air avec une table à digitaliser. Actes du 2ème congrès international de robotique pédagogique. Montréal.

8. M. TROPIS et C. BOUYSSET (1988). Quelles techniques numériques pour les acquisitions de données. Actes des 3èmes journées IPSP. Grenoble.

\section{RÉSUMÉS}

Utilisation des micro-ordinateurs dans des activités non directives en travaux pratiques de sciences physiques.

L'expérimentation a commencé au printemps 1992 juste après l'aménagement des salles nouvellement construites. Les élèves avaient déjà été un peu initiés à l'outil depuis le début de la classe de seconde lors d'expériences de cours ou de travaux pratiques collectifs. Les années suivantes, les activités pourront être pratiquées tout au long de l'année. L'expérience a été faite avec trois classes de première $S$ (jugées de niveau très moyen) durant trois séances de travaux pratiques. La première séance a été consacrée à une initiation à l'outil et les deux autres à l'étude des circuits dérivateurs.

Des observations ont pu être faites. D'abord on a pu constater que la plupart des élèves arrivent plus ou moins au résultat (même au passage au circuit intégrateur) aidés en cela par la dynamique de la classe. La remarque essentielle concerne le manque d'aptitude des élèves à l'autonomie, sans doute occasionné par une formation préalable excessivement directive. On sent qu'ils n'osent pas prendre d'initiatives même dans le fonctionnement du logiciel, comme s'ils avaient peur de le détériorer.

Aux nombreux collègues qui trouvent que la recherche-action sur l'ordinateur outil de laboratoire traîne en longueur et que le passage à l'acte se fait attendre, nous pouvons répondre que pour que ces techniques soient assimilables il faut qu'elles soient pratiquées par les élèves. 
Pour cela il faut d'abord que le matériel soit en quantité suffisante dans les collections des laboratoires, ensuite que les enseignants aient suffisamment d'imagination pour concevoir des situations qui ne se contentent pas de reproduire les démarches pédagogiques ancestrales. L'expérience du lycée de Manosque constitue dans ce domaine un modèle qui mérite d'être suivi.

Use of computers for no-directing activities in laboratory of physical sciences.

INDEX

Mots-clés : activité, ordinateur, travaux pratiques

\section{AUTEURS}

\section{TROPIS}

Direction des lycées et collèges, bureau des innovations pédagogiques

\section{BOUYSSET}

Direction des lycées et collèges, bureau des innovations pédagogiques

\section{N. CAUVIN}

Lycée Félix Esclangon de Manosque

\section{CH. SALLE}

Lycée Félix Esclangon de Manosque

A. MEGEL

Lycée Félix Esclangon de Manosque

\section{GROUPE IDEAO}

Laboratoire d'étude des méthodes modernes d'enseignement, université Paul Sabatier de Narbonne 